\title{
Study of Serum Vitamin-C levels in Carcinoma Stomach
}

\author{
Subrat kumar sahu ${ }^{1}$, Ranjana Giri ${ }^{2}$ \\ Assistant Professor ${ }^{1}$,Associate Professor ${ }^{2}$ \\ Department Of Surgery ${ }^{1}$,Department Of Pathology ${ }^{2}$,Kalinga Institute Of Medical Science. \\ KIIT,Bhubaneswar,India.
}

\begin{abstract}
:
Introduction:- Stomach cancer is the leading cause of cancer related death worldwide, hence known as CAPTAIN OF MENS DEATH.Vitamin -c is an antioxidant and inhibitor of carcinogen N-nitroso compound production in the stomach.Numerous case control studies show that higher dietary vitamin -c consumption is associated with decreased risk of gastric cancer but prospective studies are limited,particularly for blood measures of Vitamin-c.

Materials and Methods:- Study group includes 100 cases,devided in to five groups.38 confirmed cases of gastric cancers, 20 benign peptic ulcer diseases, 15 GI malignancies other than stomach,12 non GI malignancies and 15 healthy volunteers. Histological study was done from endoscopic specimen and surgically resected specimen.Serum vitamin -c level was estimated in all the cases.

Results:- Higher age group(56.89+13.8) and males(92.1\%) were the vulnerable group for cancer stomach.Plasma levels of Vit-C is significantly lower in cancer stomach cases in comparision to healthy volunteers which is statistically significant( $p$ value $<0.05)$.Plasma vit-c level of other GI malignancies and non GI malignancies are also less than healthy volunteers. Equal level of Vit $C$ both in cancer stomach and APD patients which is lower than that of control group.Consumption of vitamin $C$ rich food is protective against cancer stomach by its anticarcinogenic properties.The difference between vit-c rich food consumption between cancer stomach patient and healthy volunteers is statistically significant( $p$ value $<0.05)$.

Conclusion:-Plasma level of vit-c is significantly lower in cancer stomach in comparision to healthy volunteers. So vit-c supplimentation could be beneficial in such cases if further study could confirm relationship of vit-c with gastric cancer as to wheather it is a cause or effect of the disease.Further studies are also necessary to determine the mechanism of Vitamin $C$ action and any potential interactions with $H$ Pylori infection and smoking.
\end{abstract}

Keywords: Serum vitamin-c,stomach cancer.

\section{I.Introduction}

Less than a century ago ,gastric cancer was the most common cancer in the United states and perhaps throughout the world.Despite a marked decline in the incidence of stomach cancer,it remains the 3rd leading cause of cancer related death worldwide ${ }^{1}$ The impact is so tremendous that it has earned the sobriquet of captains of men's death.As per updated epidemiology cancer stomach it ranks fifth after lung,breast and colorectal cancer and prostate ${ }^{2}$.

Vitamin -c is an important enzyme cofactor and also has been suggested to have antiproliferative and proapoptotic roles in vitro,to inhibit the growth of $\mathrm{H}$ Pylori and to regulate the immune response towards $\mathrm{H}$ Pylori infection.But perhaps most importantly Vit-c can quench reactive oxygen species produced in the gastric environment,thus limiting free radical mediated damage in the gastric epithelium and it can scavenge nitrite, inhibiting in vitro nitrosation and the production of carcinogenic $\mathrm{N}$-nitroso compounds. Although a verity of factors may contribute to the carcinogenic process,gastric juice vitamin-c has recently attracted many reseachers ${ }^{3}$.This is not only because of the inter-relationship between vitamin-c and $\mathrm{H}$ pylori in the stomach but also because growing evidence suggests that dietary vitamin-c reduces gastric cancer risk ${ }^{4}$. It has been shown that vitamin $\mathrm{C}$ reduces the formation of $\mathrm{N}$-nitroso compounds in gastric juice and scavenges reactive oxygen metabolites in gastric mucosa. ${ }^{5}$

The diet is suspected to be a primary offender and adherence to certain culinary practices is associated with a high risk of gastric carcinoma.The presence of carcinogen such as N-Nitroso compounds(NOCS) and benzopyrene is particularly important.It has been shown that both ascorbic acid and dehydroascorbic acid can affect cell growth by altering cell proliferation and inducing cell death in various cell systems ${ }^{6,7}$.So keeping in view of the aforesaid factors it appears that Vit-C is probably the prime antioxidant present in vegetables and fruits which may prevent development of carcinoma of stomach.

Evidence from epidemiological and case control studies is mainly based on Vit-c concentration in serum or plasma but there are no direct data relating low gastric juice vitamin $\mathrm{c}$. 


\section{Material And Methods}

The present study was a prospective study and included 100 cases. The study duration was $2 \mathrm{yrs}$ in Chittaranjan national cancer institute,KOLKATA(W.B).

Total no of cases-100

study group is devided in to five groups.

Grade-A:Confirmed cases of gastric cancer $(\mathrm{n}=38)$

Grade B:Benign peptic ulcer diseases $(\mathrm{n}=20)$

GradeC:Confirmed cases of GI malignancies other than gastric cancer $(\mathrm{n}=15)$

Grade D:Confirmed cases of non GI malignancies $(n=12)$

Grade E:Healthy volunteers $(\mathrm{n}=15)$

Patients with other medical and/or surgical diseases other than the diseases mentioned above and

patients with more than one of the above diseases are excluded from the study group.

Collection of blood was done in empty stomach.Flexible endoscope was used for taking biopsy from the lesion.Histopathological study was done from endoscopic specimen and surgically resected specimen.

\section{Method Of Vitamin C Estimation}

Blood was collected, vit-c level was estimated and expressed in mg/dl.Estimation of vitamin c was done by 2-4 dinitrophenylhydrazine method.The principle of the method is that dehydroascorbic acid was coupled with 2,4 dinitrophenylhydrazine and the resulting derivative is treated with sulphuric acid to produce a newly observed red colour which is measured colorimetrically at $545 \mathrm{~nm}$.

Normal value of Plasma Vit-c- concentration-0.4-1.5mg/dl.

$-<0.2 \mathrm{mg} / \mathrm{dl}-$ deficient.

Various statistical methods have been adopted to verify the statistical significance of various data obtained from our study.All datas are described by mean,range, standard deviation.Relationships between diff parameters were determined by Chi square test.Probability value of less than 0.05 were considered significant and when $P$ value is $<0.01$, the difference is highly significant.

\section{Results And Discussion}

The study included 100 cases.The level of plasma vit-c level in proved cases of gastric cancer patients was estimated and compared with that of control groups like patients with benign gastric diseases(APD),GI malignancy other than stomach,Non GI malignancy and healthy volunteers. The mean age of presentation of cancer stomach is 56.89 years and APD,G I malignancy other than stomach and non GI malignancies were 47.85years,49.80years and 48.67years respectively.Incidence of cancer stomach is much higher in males $(92.1 \%)$ than females(7.9\%).

Mean plasma vit-c level is maximum in healthy volunteers $(1.12 \mathrm{mg} / \mathrm{dl})$ followed by that in the patients of other GI malignancy and nonGI malignancies i.e $1.08 \mathrm{mg} / \mathrm{dl}$ each and minimum in patients in carcinoma stomach and APD are $0.97 \mathrm{mg} / \mathrm{dl}$ each.The difference observed in mean plasma vit-c level between carcinoma stomach patients and healthy volunteers was found to be statistically significant that is $\mathrm{S} . \mathrm{E}=0.08, \mathrm{Z}=1.88, \mathrm{P}<0.05$. The difference observed in mean plasma vit $\mathrm{C}$ level between carcinoma stomach with GI malignancy other than stomach and non GI malignancies were also found statistically significant $(\mathrm{P}<0.05)$.It is clearly evident that that the plasma level of vit-c in cancer stomach patient is lower in comparision to healthy volunteers,other GI malignancies,nonGI malignancies.But the level of plasma vit $\mathrm{c}$ in carcinoma stomach is same as in APD group(Table-1)

Table-1. Plasma vitamin-c (mg/dl) level in study subjects

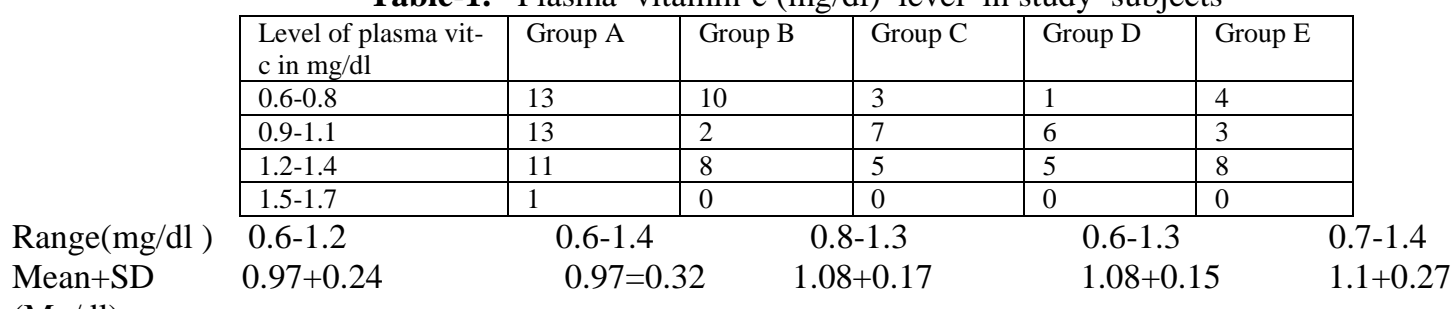

$(\mathrm{Mg} / \mathrm{dl})$

Consumption of Vit-c rich food among cancer stomach patients(31.5\%) is less in comparision to healthy volunteers(66.1\%), $\mathrm{APD}(55 \%)$ and other GI malignancy $33.3 \%)$. The difference observed in vit-c rich food consumption between healthy volunteers was found to be statistically significant $(\mathrm{p}=<0.05)$. This shows that consumption of vit-c rich food is negatively corelate with stomach cancer.(table-2) 
Table-2. Consumption of vit-c rich food among study subjects

\begin{tabular}{|l|l|l|l|l|l|}
\hline Vit-c rich food & Group A & Group B & Group C & Group D & Group E \\
\hline Consumed & $12(31.58 \%)$ & $11(55 \%)$ & $5(33.3 \%)$ & 00 & $10(66 \%)$ \\
\hline Not consumed & $26(68.4 \%)$ & $9(45 \%)$ & $1(66.7 \%)$ & 12 & $5(34 \%)$ \\
\hline
\end{tabular}

In the present study the plasma vit-c level in patients with cancer stomach is clearly less than that of healthy volunteers which is statistically significant.Equal level of vit-c both in cancer stomach and APD patients which is lower than that of control group.Consumption of vit-c rich food is protective against cancer stomach by its anticarcinogenic properties.

\subsection{Vitamin-C in health and disease}

Vitamin $\mathrm{C}$ is an acidic molecule with strong reducing activity and is an essential component of most living tissues .Vitamin $\mathrm{C}$ exerts profound effect on our health specially in the held of immune function, wound healing,cardiovascular diseases, diabetes,cataract,pulmonary function and cancer.Various studies provide evidence of protective roles of vitamin -c against various types of cancer e,g cancer of esophagus, larynx, oral cavity,pancrease,stomach, colon,rectum and breast.Ascorbic acid has been observed to reduce the binding of polycyclic aromatic carcinogens to DNA(molecular basis of carcinogenesis) and to reduce/delay tumor formation in several animal models.Ascorbic acid is also a potent inhibitor of nitrosamine-induced carcinogenesis,functioning as a nitrite scavenger.This action results from the reduction by ascorbate of nitrate to NO, thus blocking the formation of nitrosamines.

\subsection{Vitamin $\mathrm{C}$ in gastric cancer}

Vitamin $\mathrm{C}$ is thought to be an important local protective factor against gastric carcinogenesis. H Pylori infection induces marked infiltration of inflammatory cells within the gastric mucosa, These inflammatory cells such as neutrophils and monocytes synthesize and release copious amount of toxic ROMs ${ }^{7}$ which may be neutralised by vitamin $\mathrm{C}$ and other antioxidants in the stomach.It has been proposed that vitamin $\mathrm{C}$ may have a major role in preventing gastric cancer by inhibiting the formation of NOCs and neutralising the toxicity of ROMs in the stomach.However previous studies have suggested that vitamin $\mathrm{C}$ may also directly interfere with the synthesis of host cell DNA,RNA and protein,thereby inhibit cell proliferation and induce cell death ${ }^{8,9}$.

\section{IV.Conclusion}

The present study critically analysed the role of different prognostic variables in cancer stomach by taking in to account of proper history,clinical examination and relevant investigations.Higher age group and males are the vulnerable group of cancer stomach .vit-c by its antioxidant and anticarcinogenic property prevents the development of gastric cancer.Plasma level of vit-c significantly lower in cancer stomach cases in comparision to healthy volunteers but not below the deficiency $(<0.2 \mathrm{mg} / \mathrm{dl})$ level. So vit-c supplimentation could be beneficial in such cases if further study could confirm relationship of vit-c with gastric cancer as to wheather it is a cause or effect of the disease.In our study it is evident that lower levels of vit-c in plasma is seen to be closely associated with cancer stomach.But in contrary a similar trial should be done in suspected population where there is high dose of injection of vit-c should be compared with low vit-c receiving group to evaluate for presence of early gastric carcinoma as screened by upper GI endoscopy and biopsy.Further studies are also necessary to determine the mechanisms of vitamin- $\mathrm{C}$ action, any potential interactions with $\mathrm{H}$ Pylori infection and smoking.

\section{References}

[1]. "Chapter 1.1".World cancer report 2014. World health organisation.2014 ISBN 9283204298.

[2]. Ferlay J,Soerjomataram I,Ervik M,Dikshit R,Eser S,Mathers C.GLOBACON 2012 v1.1,Cancer incidence and mortality worldwide:IARC cancer Base no.11.International Agency for Research on cancer,2o14.

[3]. Reed PI .Vitamin C,Helicobacter Pylori infection and gastric carcinogenesis.Int J Vitam Nutr Res 1999;69:220-7.

[4]. Correa P,Malcom G,Schmidt B,et al.Review article:Antioxidant micronutrients and gastric cancer.Aliment Pharmacol Ther 1998;12(suppl 1):73-82.

[5]. Zhang ZW,Farthing MJG. Helicobacter pylori and gastric malignancy:Importance of oxidants,antioxidants and other cofactors.In:Hunt RH,Tytgat GNJ,eds.Helicobacter pylori:Basic mechanisms to clinical cure 2000.London:Kluwer Academic Publishers, 2000:513-24.

[6]. Brrigelius-Flohe R ,Flohe L Ascorbic acid,cell proliferation, and cell differentiation in culture.Subcell Biochem 1996;25:83-107.

[7]. Sakagami H,Satah K.Modulating factors of radical intensity and cytotoxic activity of ascorbate.Anticancer Res 1997;17:3513-20.

[8]. Conner EM.Grisham MB.Inflammation,free radicals and antioxidants .Nutrition 1996;12:274-7.

[9]. Lupulescu A.Vitamin C inhibits DNA,RNA and protein synthesis in epithelial neoplastic cells.Int J VItam Nuir Res 1991;61:125-9.

[10]. Lupulescu A .Ultrastructure and cell surface studies of cancer cells following Vit C administration.Exp Toxicol Pathol 1992;44:39. 